\title{
f2MOVE: fMRI-compatible haptic object manipulation system for closed-loop motor control studies
}

\author{
Anastasia Sylaidi ${ }^{1,4}$, Pedro Lourenco ${ }^{1,4}$, Sathiji Nageshwaran ${ }^{3,4,5}$, Chin-Hsuan Lin $^{1}$, \\ Marisol Rodriguez ${ }^{1}$, Richard Festenstein ${ }^{3,4,5}$ \& A. Aldo Faisal ${ }^{1,2,4}$, Member IEEE
}

\begin{abstract}
Functional neuroimaging plays a key role in addressing open questions in systems and motor neuroscience directly applicable to brain machine interfaces. Building on our low-cost motion capture technology (fMOVE), we developed f2MOVE, an fMRI-compatible system for 6DOF goaldirected hand and wrist movements of human subjects enabling closed-loop sensorimotor haptic experiments with simultaneous neuroimaging. f2MOVE uses a high-zoom lens high frame rate camera and a motion tracking algorithm that tracks in real-time the position of special markers attached to a hand-held object in a novel customized haptic interface. The system operates with high update rate $(120 \mathrm{~Hz})$ and sufficiently low time delays $(<20 \mathrm{~ms})$ to enable visual feedback while complex, goaloriented movements are recorded. We present here both the accuracy of our motion tracking against a reference signal and the efficacy of the system to evoke motor control specific brain activations in healthy subjects. Our technology and approach thus support the real-time, closed-loop study of the neural foundations of complex haptic motor tasks using neuroimaging.
\end{abstract}

\section{INTRODUCTION}

Neuroscience has systematically studied sensorimotor functions for more than 100 years. Common methodological approaches have involved the use of psychophysical experiments and computational theories of inference and policy formation [1],[2],[3],[4],[5],[6]. The latter provide insight into the patterns of adaptive responses in tasks that introduce target, workspace or force-field perturbations [7],[8] and examine multisensory integration [9], body and world representation [10] or performance optimization in the face of sensory and motor noise [11].

However, despite this substantial progress in the investigation of motor behavior, less advancement has been achieved in associating motor psychophysics and computational models of sensorimotor control to their underlying neural foundation. A growing number of studies have attempted to address this challenge with the use of fMRI technology,

Brain \& Behaviour Lab - ${ }^{1}$ Dept. of Bioengineering \& ${ }^{2}$ Dept. of Computing, Imperial College London, South Kensington Campus, SW7 2AZ, London, UK, ${ }^{3}$ Gene control mechanisms \& disease group, Division of Brain Sciences, Faculty of Medicine, Imperial College London, ${ }^{4}$ Imperial Clinical Research Facility, Imperial Centre for Translational and Experimental Medicine, W12 0NN London, UK, ${ }^{5}$ MRC Clinical Sciences Centre, Hammersmith Hospital Campus, W12 0NN, London, UK - a.faisal at imperial.ac.uk

*This work was supported by a Colin Caro Bioengineering Fellowship, a scholarship from the Foundation for Education and European Culture (AS), a Wellcome Trust-GSK Fellowship to SN (No. WT103456MES), Fellowship of the Taiwanese Ministry of Education (1001163-2-UK-003) to CHL and a NIHR/BRC Rare Diseases grant (RF \& AAF). The authors also wish to thank Albert L. Busza and Julie A. Fitzpatrick for their valuable support in undertaking fMRI sessions at the C.I.F. of Hammersmith Hospital, London. which they primarily employed during lab-constrained simple hand reaching movements or non-specific open-loop manipulations (e.g. finger tapping). The main reason for this restriction lies in the technical constraints of fMRI, which is often incompatible to advanced motion tracking systems, that could monitor more complex motor behavior.

Here we designed and developed, f2MOVE, a novel 6DOF fMRI-compatible motion tracking system to support realistic object manipulation (haptic) tasks during a neuroimaging session. The development was motivated by a rapidly growing body of studies that focuses on life-like tasks, which enable movements in naturalistic settings without the usual confines of strict lab protocols. This work supports a better understanding of human natural movement statistics [12],[13], provides insight into the structure of motor primitives and thus carries, through movement predictability, direct implications for neuroprosthetic approaches and brainmachine interfaces [14].

f2MOVE was built upon our low-cost motion capture technology, fMOVE [15], which we expanded to adjust to the fMRI environment (Clinical Imaging Facility, Hammersmith Hospital, London). We complemented our system with a methodological platform, which can support closed-loop task contexts to encourage learning based on online sensory feedback of performance. f2MOVE's motion

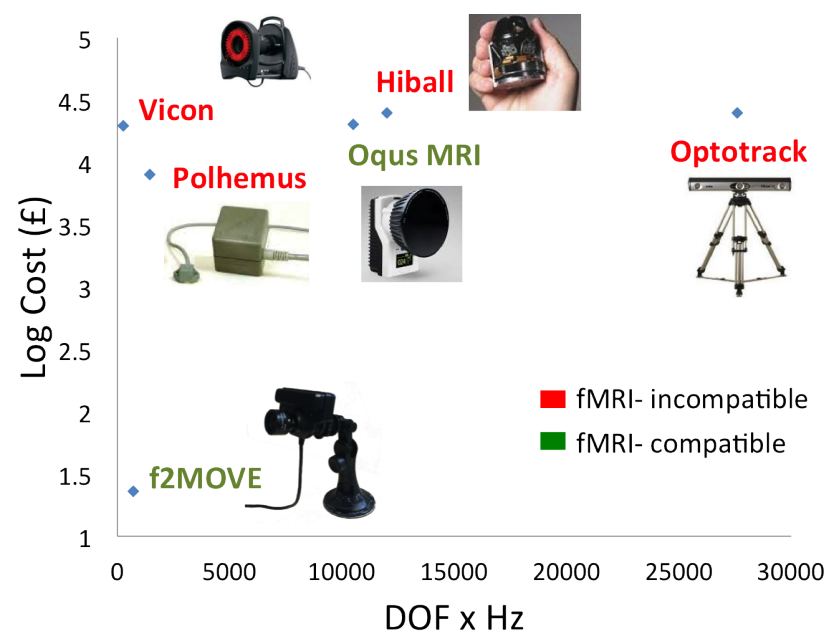

Fig. 1. Frugal innovation for motion tracking: Cost versus efficiency of established motion tracking systems (not fMRI compatible) against which f2MOVE (fMRI compatible) is compared. f2MOVE corresponds to the lowest cost level (camera expenses) and possesses satisfactory motion tracking performance within the range of state-of-the-art motion trackers (e.g. Polhemus, Vicon). 

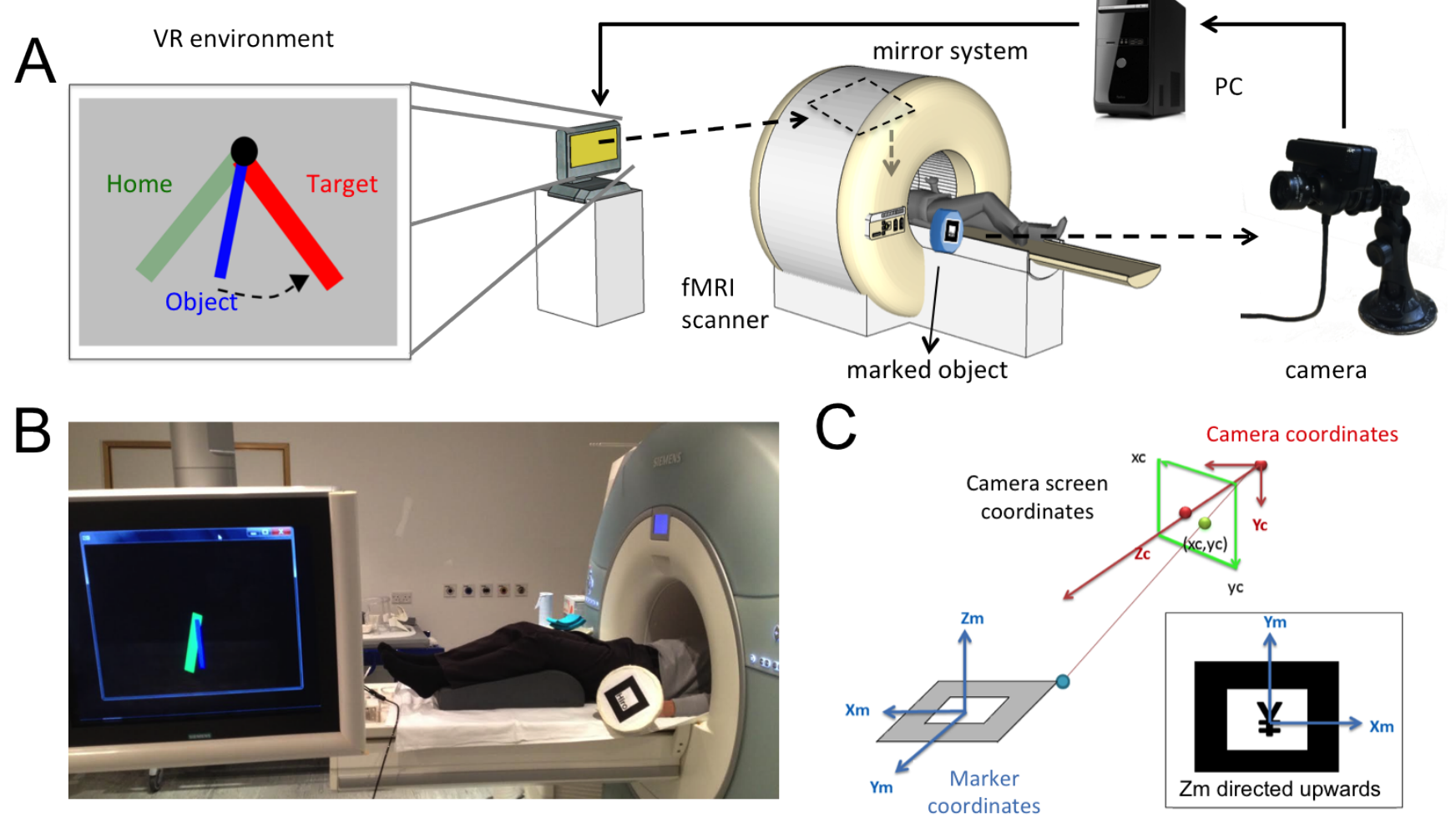

Fig. 2. Experimental setup: (A) displays the system's components which interact during an actual experimental session (B) to support closed-loop haptic object manipulation experiments. The object position is tracked by our customized marker-based motion tracker, which provides a PC-run software module with continuous information about the subject 's movements. The software platform displays a virtual online feedback of the monitored behavior and performance, which is in turn presented to the subject via a computer screen and a mirror system adjusted to the scanner. (C) The marked object position is estimated based on transformations between a marker-based $\left(X_{m}, Y_{m}, Z_{m}\right)$ and a camera-based $\left(X_{c}, Y_{c}, Z_{c}\right)$ coordinate system.

tracking performance lies in the same range as established fMRI-incompatible motion tracking methods (e.g. in Fig. 1 Vicon with 1DOF motion tracking at $250 \mathrm{~Hz}$, Polhemus Liberty with 6DOF at $240 \mathrm{~Hz}$ ). Furthermore, it presents a significant cost-efficiency benefit compared to other motiontracking technologies with advanced features (e.g. Hiball with 6DOF motion tracking at $2000 \mathrm{~Hz}$, Optotrack with 6DOF at $4600 \mathrm{~Hz}$, Oqus MRI with 6DOF at $1750 \mathrm{~Hz}$ ).

\section{Methods}

\section{A. Hardware}

Our system allows a closed-loop interaction of human subjects lying inside a 3T fMRI scanner and a PC-handled experimental paradigm (Fig. 2 A,B). Subjects use their dominant hand to hold and manipulate a compact object so as to move it correctly between some instructed home and target orientation. The exact task conditions are displayed to them via a mirror system built inside the scanner, which reflects the virtual progression of the task on a computer screen. Motor performance is tracked by a high-zoom lens, low cost camera (Playstation 3 Eye) positioned on one of the scanner room walls, at a $3.5 \mathrm{~m}$ outside of the scanner, facing the foot-end view of the cylinder. The camera operates at a $120 \mathrm{~Hz}$ frame-rate for a $320 \times 240$ pixel resolution with low time delays $(<20 \mathrm{~ms})$ and can track 6DOF movement of the object, based on a customized 2D marker adjusted on the latter. We mounted it on a customized platform that allows a $6 \mathrm{DOF}$ rotation and precice alignment of the camera orientation with the scanner opening. The motion tracking system feeds all acquired images into a PC-based software, module, which monitors motor behavior and determines the transition between consecutive experimental phases. This module communicates with the subject via the scanner-based mirror system, on which it provides visual access to the task goals, and real-time feedback of motor performance.

\section{B. Continuous closed-loop object manipulation}

The camera continuously records images, which support the estimation of the object position and the visualization of its virtual analogue based on a software library for designing Augmented Reality applications (ARToolkit). In particular, f2MOVE estimates the object position, based on two coordinate systems which are related via an affine transformation (Fig. 2 C): a camera based (3D) and a marker based (3D). The marker based $X_{m}$ and $Y_{m}$ axes are aligned with the horizontal and vertical marker sides respectively, while $Z_{m}$ points away vertically from the $2 \mathrm{D}$ plane defined by $X_{m}$ and $Y_{m}$. The marker center is located at $\left(X_{m}, Y_{m}, Z_{m}\right)=(0,0,0)$. The module is designed based on a custom made motorlib $C$ code library and opengl and operates by defining consecutive experimental stages as finite states which allow transition, depending on the monitored motion information. 

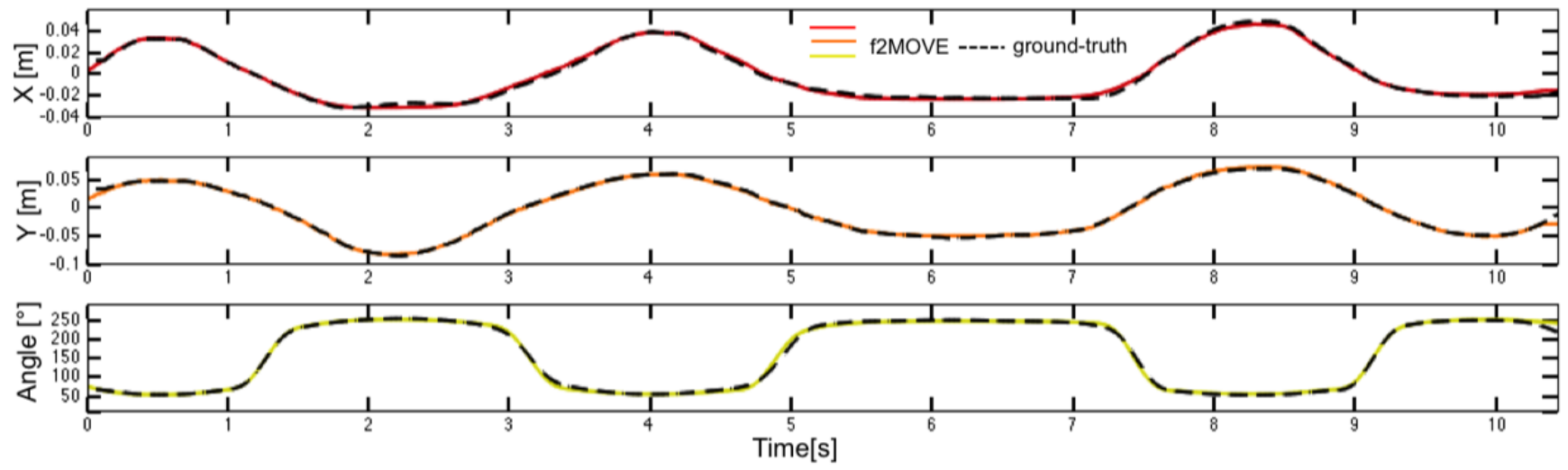

Fig. 3. Testing the tracking accuracy: We compared the f2MOVE signal against the signal monitored by Optitrack Flex-13, which was used as reference. Both signals were acquired simultaneously in a lab setting simulating the experimental conditions of an object manipulation paradigm designed for clinical studies inside the fMRI environment. The f2MOVE signal demonstrates a satisfactory matching to the reference motion tracking performance (z-signal not included since movement in this axis is minimal).

We developed a novel haptic interface that includes a light fMRI-compatible object. The design consists of a plastic handle, used as the reception for mounting multifaceted objects of variable shape and dynamics. For the needs of our first clinical sessions we designed and used an object with thin cylindrical body that hosted a single marker tractable by our motion tracking system (Fig. 2 B). Depending on the needs of the experimental study and the complexity of the examined movement, the number of markers on the tracked object can be increased to ensure that occasionally obscured markers, are substituted by more visible ones. This offers flexibility to the experimental design and allows us to investigate motor behavior during haptic interaction with complex objects.

\section{Tracking accuracy}

We tested the tracking accuracy of f2MOVE against a standardized motion tracking system (Optitrack Flex-13). In particular, our reference is a state-of-the-art infrared markertracking system that offers millimiter resolution of 3D spatial displacements and operates with accuracy at $100 \mathrm{~Hz}$. In order to compare the signal acquired by the two systems, we aligned the f2MOVE camera with one of the three used Optitrack cameras. We placed 4 Optitrack markers at the corners of the f2MOVE 2D marker, so as to create a rigid body with the same center to f2MOVE's marker center. We acquired motion information from a healthy female subject, based on an experimental paradigm designed for the Clinical Imaging Facility at Hammersmith Hospital, London. In this paradigm the subject continuously moves the marked object between an instructed home and target orientation.

After data acquisition, the f2MOVE signal was downsampled to match the reference signal. The two signals were subsequently aligned temporally by matching the first trial initiation after a resting period. A mean affine transformation was estimated for resting period data, to match the f2MOVE signal to the reference signal, via rotation and translation.
The transformed signals we compared based on RMSE and $R^{2}$ to determine f2MOVE tracking accuracy.

\section{RESULtS}

We tested the operating features and tracking accuracy of our system, f2MOVE, in lab conditions and inside the fMRI environment to establish its utility for closed-loop object manipulation tasks. f2MOVE operates successfully at $120 \mathrm{~Hz}$ frame-rate with low time-delays and tracks 6DOF movement of the marked object.

f2MOVE's precise tracking accuracy was estimated against an established infrared marker-tracking system (Optitrack Flex-13) in a lab setting simulating the current experimental setting of our system at the Clinical Imaging Facility at Hammersmith Hospital, London. We measured motion information for a task instructing the manipulation of the experimental object inside a specified orientation range. We aligned the two acquired signals in the temporal and spatial domain to achieve matching, after which we estimated their RMSE difference. Fig. 3 reveals good consistency of movement measurements between f2MOVE and our reference system, both in the 2 dimensions used for our experimental paradigm (the execution was instructed on a $2 \mathrm{D}$ xy plane and movement in the $\mathrm{z}$ dimension was minimal) and in the angle rotation domain of the xy plane. The matching between signals was estimated at $R^{2}=0.98$ and $R M S E=2 \mathrm{~mm}, R^{2}=$ 0.99 and $R M S E=2.6 \mathrm{~mm}, R^{2}=0.99$ and $R M S E=1.02 \mathrm{~mm}$, $R^{2}=0.99$ and $R M S E=4.2^{\circ}$ for the $\mathrm{x}, \mathrm{y}, \mathrm{z}$ translation and rotation of the xy plane around the z-axis respectively.

Our tested system was transferred and adjusted to the fMRI setting at the Clinical Imaging Facility at Hammersmith Hospital, London. It was used to support scanning sessions on healthy subjects during an object manipulation paradigm. Fig. 4.A illustrates 3D rendered views of activation patterns for task-versus-rest conditions in our paradigm. These views reflect the enhanced role of specific brain areas (e.g. cerebellum, SMA) in error-driven closed-loop object 

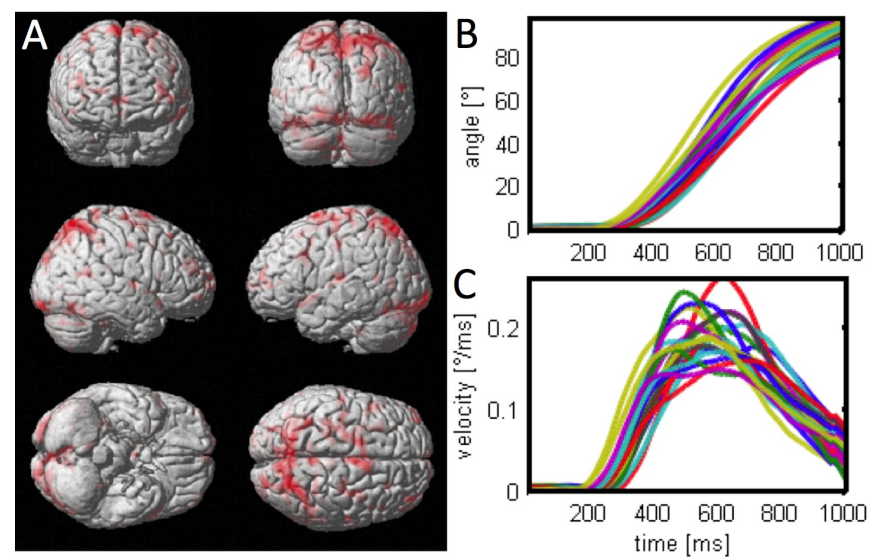

Fig. 4. Linking neuroimaging and motor psychophysics: f2MOVE enabled fMRI sessions on a healthy subject during a closed-loop object manipulation task, equivalent to the one tested in our simulated lab conditions (moving object between home and target orientation). (A) Our methodological platform allowed the acquisition of cortical activation patterns in taskversus-rest conditions. An examination of these patterns reveals the role of the cerebellum and SMA in error-driven motor learning. Network-level analysis can support a further understanding of the neural implementation of motor behavior in complex naturalistic manipulations. (B) The object orientation and $(\mathrm{C})$ angular velocity over time (trial start at $0 \mathrm{~ms}$ ). across trials can be examined in parallel with the cortical activation patterns, so as to determine behavioral performance measures that can be subsequently employed as regressors against the fMRI signal.

manipulations. Furthermore, the simultaneously acquired behavioral data (Fig. 4.B,C) can be regressed against activation patterns to examine neural correlates of behaviorally determined motor control mechanisms. Such approaches can shed further light into the foundation of sensorimotor functions in naturalistic motor tasks, such as the one examined by our system.

\section{CONCLUSION}

We designed and developed an fMRI-compatible haptic object manipulation system (f2MOVE) for closed-loop 6 DOF motor control studies. We built upon our previously developed 3 DOF marker-based motion tracking system to adjust our technology to the fMRI environment and to expand it so as to accommodate motor experiments with goaldirected hand and wrist movements as well as the interaction with objects of variable dynamics.

f2MOVE poses technical benefits for high frequency data acquisition inside the fMRI environment, which is commonly incompatible to most currently established motion tracking systems. Moreover, building on our previous experience on low-cost wearable kinematic body-sensor networks [16], we aimed at developing an easily affordable neurotechnological tool. Its cost is limited to the customized camera price, in contrast to the expences involved in fMRI-compatible motion tracking approaches which rely on a robotic manipulandum for motion detection and/or perturbation [17],[18]. f2MOVE, thus offers an accessible technological and methodological platform to re-approach the objectives of motor neuroscience in examining the neural foundation of sensorimotor control and learning. It also supports the design of clinicallyvaluable behavioral and neuroimaging markers to monitor motor coordination in healthy or pathological cases (e.g. neurodegenerative diseases).

\section{REFERENCES}

[1] D. M. Wolpert, J. Diedrichsen, and J. R. Flanagan, "Principles of sensorimotor learning," Nat. Rev. Neurosci., vol. 12, no. 12, pp. 739$751,2011$.

[2] E. Todorov, "Optimality principles in sensorimotor control," Nat. Neurosci., vol. 7, no. 9, pp. 907-915, 2004.

[3] S. H. Scott, "Optimal feedback control and the neural basis of volitional motor control," Nat. Rev. Neurosci., vol. 5, no. 7, pp. 532546, 2004.

[4] K. J. Friston, J. Daunizeau, and S. J. Kiebel, "Reinforcement learning or active inference?" PloS one, vol. 4, no. 7, p. e6421, 2009.

[5] K. Friston, "The free-energy principle: a unified brain theory?" Nat. Rev. Neurosci., vol. 11, no. 2, pp. 127-138, 2010.

[6] K. P. Körding and D. M. Wolpert, "Bayesian decision theory in sensorimotor control," Trends Cogn. Sc., vol. 10, no. 7, pp. 319-326, 2006.

[7] R. Shadmehr and F. A. Mussa-Ivaldi, "Adaptive representation of dynamics during learning of a motor task," J. Neurosci., vol. 14, no. 5, pp. 3208-3224, 1994.

[8] R. Shadmehr and Z. M. Moussavi, "Spatial generalization from learning dynamics of reaching movements," J. Neurosci., vol. 20, no. 20, pp. 7807-7815, 2000.

[9] S. Vaziri, J. Diedrichsen, and R. Shadmehr, "Why does the brain predict sensory consequences of oculomotor commands? optimal integration of the predicted and the actual sensory feedback," J. Neurosci., vol. 26, no. 16, pp. 4188-4197, 2006.

[10] A. Sylaidi and A. A. Faisal, "What is the hierarchical representation of tasks involving objects with complex internal dynamics?" Front. Comput. Neurosc., vol. Bernstein Conference, 2012.

[11] A. A. Faisal, L. P. Selen, and D. M. Wolpert, "Noise in the nervous system," Nat. Rev. Neurosci., vol. 9, no. 4, pp. 292-303, 2008.

[12] J. N. Ingram, K. P. Körding, I. S. Howard, and D. M. Wolpert, "The statistics of natural hand movements," Exp. Brain Res., vol. 188, no. 2, pp. 223-236, 2008.

[13] I. S. Howard, J. N. Ingram, K. P. Körding, and D. M. Wolpert, "Statistics of natural movements are reflected in motor errors," $J$. Neurophysiol., vol. 102, no. 3, pp. 1902-1910, 2009.

[14] A. A. Thomik, D. Haber, and A. A. Faisal, "Real-time movement prediction for improved control of neuroprosthetic devices," in Neural Engineering (NER), 2013 6th International IEEE/EMBS Conference on. IEEE, 2013, pp. 625-628.

[15] M. Rodriguez, A. Sylaidi, and A. A. Faisal, "Developing a novel fmri-compatible motion tracking system for haptic motor control experiments," in Neurotechnix, 2014, 2014.

[16] C. Gavriel and A. A. Faisal, "Wireless kinematic body sensor network for low-cost neurotechnology applications in-the-wild," in Neural Engineering (NER), 2013 6th International IEEE/EMBS Conference on. IEEE, 2013, pp. 1279-1282.

[17] J. Diedrichsen, Y. Hashambhoy, T. Rane, and R. Shadmehr, "Neural correlates of reach errors," J. Neurosci., vol. 25, no. 43, pp. 99199931, 2005

[18] S. Menon, M. Yu, K. Kay, and O. Khatib, "Haptic fmri: Accurately estimating neural responses in motor, pre-motor, and somatosensory cortex during complex motor tasks," in Engineering in Medicine and Biology Society (EMBC), 2014 36th Annual International Conference of the IEEE. IEEE, 2014, pp. 2040-2045. 